\title{
BMJ Open Comparative study of treatment interventions for patellar tendinopathy: a protocol for a randomised controlled trial
}

\author{
Maria Pilar López-Royo, ${ }^{1,2}$ Eva Maria Gómez-Trullén, ${ }^{3}$ Maria Ortiz-Lucas, ${ }^{1}$ \\ Rita Maria Galán-Díaz, ${ }^{1}$ Ana Vanessa Bataller-Cervero, ${ }^{1}$ Zaid Al-Boloushi, , ,2,4 \\ Yasmina Hamam-Alcober, ${ }^{1}$ Pablo Herrero (D) ${ }^{1}$
}

To cite: López-Royo MP, Gómez-Trullén EM, OrtizLucas M, et al. Comparative study of treatment interventions for patellar tendinopathy: a protocol for a randomised controlled trial. BMJ Open 2020;10:e034304. doi:10.1136/ bmjopen-2019-034304

- Prepublication history and additional material for this paper are available online. To view these files, please visit the journal online (http://dx.doi. org/10.1136/bmjopen-2019034304).

Received 13 September 2019 Revised 21 January 2020 Accepted 23 January 2020

Check for updates

(C) Author(s) (or their employer(s)) 2020. Re-use permitted under CC BY-NC. No commercial re-use. See rights and permissions. Published by BMJ.

For numbered affiliations see end of article.

Correspondence to

Dr Pablo Herrero;

pherrero@usj.es

\section{ABSTRACT}

Introduction Patellar tendinopathy is a degenerative disease of the patellar tendon, which affects athletes from a variety of sports, and is especially predominant in sports involving high-impact jumping. The aim of this study is to determine the additional effect of two interventions combined with eccentric exercise and compare which one is the most effective at short-term and long-term follow-up for patients with patellar tendinopathy.

Methods and analysis This study is a randomised controlled trial with blinded participants. Measurements will be carried out by a specially trained blinded assessor. A sample of 57 patients with a medical diagnosis of patellar tendinopathy will participate in this study and will be divided into three treatment groups. Eligible participants will be randomly allocated to receive either: (a) treatment group with percutaneous needle electrolysis, (b) treatment group with dry needling or (c) treatment group with placebo needling. In addition, all groups will perform eccentric exercise. Functionality and muscle strength parameters, pain, ultrasound appearances and patient perceived quality of life shall be evaluated using the Victorian Institute of Sports Assessment for patellar (VISA-P), jump tests, Visual Analogue Scale, ultrasound images and Short Form-36 (SF-36), respectively. Participants will be assessed at baseline, at 10 weeks and at 22 weeks after baseline. The expected findings will allow us to advance in the treatment of this injury, as they will help determine whether a needling intervention has additional effects on an eccentric exercise programme and whether any of the needling modalities is more effective than the other.

Ethics and dissemination This protocol has been approved by the Ethics Committee of Aragon ( $\mathrm{N}^{\circ}$ PI15/0017). The trial will be conducted in accordance with the Declaration of Helsinki.

Trial registration number NCT02498795

\section{INTRODUCTION}

Patellar tendinopathy (PT), also known as jumper's knee, is a degenerative condition affecting the patellar tendon resulting in anterior knee pain associated with focal and palpable tenderness at the inferior pole of the
Strengths and limitations of this study

- This randomised clinical trial will report the effects on functionality and pain of three different treatment interventions in both the short-term and long-term.

- The double-blinded and placebo-control design will enhance objectivity and help reduce bias.

- The effects of two minimally invasive treatments in physical therapy will be compared for the first time in patellar tendinopathy.

patella. This disorder has similar histological findings to other tendon disorders characterised by an increased thickness of the tendon and changes in vascularity and cellularity, with incompletely healed tendon microruptures and disturbed collagen distribution. ${ }^{1}$

This degenerative condition affects athletes from a variety of sports, and is especially predominant in sports involving high-impact jumping. The overall prevalence of PT in non-elite players is $8.5 \%$, although this figure increases in sports that place high demands on the patellar ligament, increasing up to $14.2 \%$ in volleyball athletes. Among elite volleyball and basketball players, a prevalence of $45 \%$ and $32 \%$, respectively, has been reported. In addition, jumper's knee is almost twice as common among male non-elite athletes when compared with female athletes. ${ }^{2}$

The diagnosis is typically based on the clinical history and symptomatic findings. Currently, imaging techniques, such as colorDoppler ultrasound (CD-US) and grey scale ultrasound (GS-US) can be used for the assessment of the patellar tendon to clinically confirm the diagnosis. ${ }^{3}$

Treatments used for PT fall into two major groups. The first group comprises medical treatments which include non-steroidal antiinflammatory drugs (NSAIDs), platelet-rich 
plasma (PRP) injection ${ }^{4}$ and autologous growth factors. ${ }^{5}$ The second group consists of physical therapies, including both conservative and invasive approaches (needling techniques).

Conservative therapies are generally accepted as the first line of approach for managing $\mathrm{PT},{ }^{67}$ considering exercise as the gold standard of treatment, either eccentric exercise (EE) or high slow resistance training programmes. Both have demonstrated similar effectiveness in the treatment of PT. ${ }^{6-8}$ In 2012, EE was shown to be effective in the treatment of tendinopathies at various locations of the body, including PT, with a greater likelihood of clinical improvement when performed on a declined surface. ${ }^{689}$ In recent years, further evidence now supports the fact that exercise is more effective than other conventional treatments in tendinopathy, such as iontophoresis, ultrasound (US), Cyriax treatment, etc. ${ }^{10}$

Physical therapy approaches for PT continue to evolve and a number of innovative treatment options are now available, such as dry needling (DN),${ }^{11}$ electrotherapeutic invasive modalities (eg, electrolysis) ${ }^{12-14}$ and extracorporeal shockwave therapy ${ }^{15}$ Recently, research has focussed on regenerative therapies with high expectations of success because some of these techniques seem to achieve a rapid regeneration of the injured tendon. ${ }^{11216}$ However, evidence-based regenerative therapies are limited and there is no agreement to date regarding which of these is the most effective. ${ }^{17} \mathrm{DN}$ consists of the insertion of a needle (filiform and solid, non-bevelled) with the aim of provoking a local injury leading to an inflammatory response and the subsequent regeneration of the injured area in approximately 1 week. A study performed by Abat et al reported that DN induced histological and mechanical changes in rat Achilles tendons at week 1, with changes persisting at week $4 .{ }^{18}$ Percutaneous needle electrolysis (PNE) is an ultrasound-guided technique used by physiotherapists consisting of causing localised lysis in the damaged and/or degenerated tissue by means of a galvanic current transmitted through an acupuncture needle. This technique may affect inflammatory mediators in damaged muscle tissue and influence the new vascularisation of the injured area in rats. ${ }^{18}$ James et al ${ }^{1}$ carried out a cohort study in humans analysing one group treated with DN and another treated with autologous blood injections. In both cases, they found improvements compared with the baseline measurements. However, this study failed to find differences between the different treatments, concluding that both techniques were equally effective. In relation to PNE, a former study ${ }^{14}$ analysed the treatment effect of electrolysis applied once a week in a group of patients without any control or comparative group, reporting that patients obtained statistically and clinically significant improvements compared with baseline measurements.

From a biological point of view, it seems reasonable to hypothesise that a patient will obtain benefits thanks to the mechanical effects provided by the needle, ${ }^{16}$ and that patients may benefit more if the electrolysis effect is added to the mechanical stimuli provided by the needle. ${ }^{19}$
Therefore, the aim of this study is to determine the additional effect of two interventions combined with EE and compare which one is the most effective at short-term and long-term follow-up for patients with PT.

\section{METHODS AND ANALYSIS Study design}

The trial is designed as a randomised, controlled, participant, investigator and outcomes assessor blinded, experimental study, aimed at comparing three different physiotherapy protocols applied in three intervention groups of PT patients. Randomisation will be performed as block randomisation with a 1:1:1 allocation.

This protocol follows the standards of the Helsinki Convention of good clinical practices. The Ethics Committee of Aragon (CEICA) has evaluated the project and has given its favourable opinion and support, $\mathrm{N}^{\circ}$ PI15/0017 (online supplementary appendix 1).

The study has been carried out following the Standard Protocol Items: Recommendations for Interventional Trials (SPIRIT) statement for clinical trial protocol and a SPIRIT Checklist has been included (online supplementary appendix 2).

\section{Study setting}

After reviewing the literature and observing the high incidence of this pathology in amateur young adult athletes who perform sports and more specifically, jump sports, patient recruitment has been performed in basketball, football, volleyball, CrossFit and handball sports clubs, together with running clubs and several gyms located in the city. A decision was made to conduct the study in Villanueva de Gallego (Zaragoza), where San Jorge University is located, as well as the laboratory to be used for assessments and treatments.

The assessments will be conducted at the Motion Analysis laboratory of San Jorge University, and the treatment will be performed at two different sites depending on the availability of both spaces and of schedules. Nonetheless, the same material will always be used.

\section{Participants}

\section{Inclusion criteria}

Participants eligible for inclusion in this study must meet the following criteria: (1) History of PT and anterior knee pain located on the inferior pole of the patella for over 3 months, (2) Aged between 18 and 45 years, (3) Palpation tenderness of the superior insertion of the patellar tendon, (4) A score below 80 on the Victorian Institute of Sports Assessment for PT (VISA-P) questionnaire.

\section{Exclusion criteria}

Exclusion criteria for the study are: (1) Knee surgery within the previous 6 months, (2) Chronic joint diseases, (3) Corticosteroid injection in the patellar tendon within the previous 3 months, (4) Contraindications 
for needling, (5) Use of drugs 48 hours previously (eg, NSAIDs), (6) Any other concomitant treatment for PT.

\section{Methodology}

In the first session, all participants will be instructed on how to perform a daily home programme of EE. This will consist of performing three sets of 15 single leg squat repetitions on a decline board every day, according to Alfredson's protocol ${ }^{20}$ increasing the speed if participants do not have pain. Participants will be informed that exercise is allowed to reach 5 in a numerical pain rating scale, ${ }^{21}$ and if it is higher then they will stop and notify the researcher, attempting once again 24 hours later following the same rules.

For the interventions, the participants will be placed in a supine position with a pillow under the knee (approximately $20^{\circ}$ of knee flexion). The area will be cleansed with an antiseptic solution (70\% Propan-2-ol, Skin-des). An ultrasound probe cover will be used during the intervention for infectious control. To determine the relevant treatment area, two factors will be considered: (1) Palpation of the areas exhibiting higher sensitivity and that reproduce the patient's symptoms, (2) Tendon areas showing degenerative changes assessed under ultrasound. Each group will receive a total of four sessions distributed throughout 8 weeks of treatment, once every 2 weeks.

\section{DN intervention combined with EE and PNE intervention combined with $\mathrm{EE}$}

Specific DN needles will be used during needling treatments, (Agu Punt, Spain). Considering the thickness of the tendon and the approach, we shall use needles measuring $0.25 \times 25 \mathrm{~mm}$. The procedure will be guided by US to ensure the specificity of application on the injured area and to guarantee that the procedure is safe for the patient. The DN needle will reach the relevant treatment area (areas with degenerative PT changes). Each session will consist of three needle insertions lasting 3 seconds each. In percutaneous needle electrolysis group (PNE-G) applications, an intensity of $3 \mathrm{~mA}$ galvanic current will be used during the 3 seconds that the procedure lasts. ${ }^{19}$ The dose of $3 \mathrm{~mA}$ has demonstrated to be as effective as $6 \mathrm{~mA}$ in the treatment of tendinopathy injuries in animal models. ${ }^{1819}$ In humans, a study conducted in 2016 showed that a dose of $3 \mathrm{~mA}$ in PT generated structural changes compatible with tendon regeneration, together with improvement of functionality and pain. ${ }^{22}$ In contrast, the same study found that lower doses were effective only for the improvement of functionality and pain. As a result, a $3 \mathrm{~mA}$ dose was selected for this study.

\section{Control group}

A sham needle will be placed on the treatment zone, simulating the same procedure as the rest of participants enrolled in the other groups. The needle will be placed in a specific holder and will be manipulated during the intervention to simulate a real treatment. This holder will have a cover over the bottom part of the same in order to avoid the needle contacting the skin.

\section{Outcomes}

Baseline data

Baseline data will include gender, age, height, weight, body mass index, affected side, level, sports and frequency of physical activity, duration of symptoms, medication and previous rehabilitation treatments and infiltrations received. A blinded observer will assess all participants at baseline, 10 weeks and 22 weeks after baseline. Participants will be asked to inform the researchers if there were any changes in medication or if they are receiving any other treatment or infiltration during the study.

\section{Primary outcome measure}

Participants will complete the VISA-P questionnaire at baseline. The VISA-P questionnaire is designed to measure the severity of PT. ${ }^{23}$ The VISA-P score is the primary outcome variable. This scale consists of eight questions, the first six questions of which employ an analogical visual scale in order to assign a score of 0 to 10 , where 10 represents the optimum state, for the purpose of quantifying pain and function in different activities, whereas the last two questions assess the level of functionality and ability to perform physical activity.

\section{Secondary outcome measure}

During the first evaluation, participants will complete the Visual Analogue Scale (VAS), considering the level of pain they feel while practicing their sport's activity. Participants will be explained that a score of 0 indicates the absence of pain whereas a score of 10 represents the maximum tolerable pain. They will also complete a questionnaire to assess their quality of life (Short Form-36 questionnaire (SF-36)). ${ }^{24}$

In order to assess tendon structure, an US evaluation using ultrasound equipment (Logiq S7 Expert, General Electric Healthcare) and a linear probe (MLG-15 5 to $10 \mathrm{MHz}$ ) will be used. The ultrasonographic assessment protocol will be carried out according to the Musculoskeletal Ultrasound Technical Guidelines: Knee, defined by the European Society of Musculoskeletal Radiology. ${ }^{25}$ The ultrasonographic assessment will consist of a longitudinal sequence from the tendon origin to the insertion and transverse sections on the pole of the patella, the tendon body and its insertion on the tibial tuberosity, with the subject in supine position, with $20^{\circ}$ knee flexion and a pillow under the knee. The presence of degenerative signs compatible with the medical diagnosis of PT (thickness of the tendon, hypoechoic areas, irregularities affecting the cortical bone, calcifications) that could be relevant for the selection of the target area will also be assessed. In addition, CD-US assessment will be carried out to detect the presence of hypervascularisation, with the subject in supine position and with the knee relaxed in full extension, in order to obtain further information to specifically define the target area. 
Table 1 Jump test's protocol

\begin{tabular}{|c|c|}
\hline \multicolumn{2}{|l|}{ Jump test protocol } \\
\hline 5 min warm up consisting & of steady jogging on a treadmill \\
\hline $\begin{array}{l}\text { Dynamic stretches lasting } \\
5 \text { min, as instructed by } \\
\text { the physical therapist }\end{array}$ & $\begin{array}{l}\text { Psoas } \\
\text { Quadriceps } \\
\text { Gluteus maximus } \\
\text { Gastrocnemius } \\
\text { Hamstring muscles }\end{array}$ \\
\hline $\begin{array}{l}\text { Three jump tests are } \\
\text { performed } \\
\text { Three jumps off the } \\
\text { ground for three times } \\
\text { for the patient to } \\
\text { become familiar with } \\
\text { the tests } \\
\text { The subject is placed } \\
\text { on the platform and } \\
\text { asked to perform each } \\
\text { test three times, with } \\
60 \text { s rest between the } \\
\text { different tests }\end{array}$ & $\begin{array}{l}\text { Abalakov test } \\
\text { Countermovement jump test } \\
\text { Squat jump }\end{array}$ \\
\hline
\end{tabular}

The highest jump is selected for the study.

On completion of the evaluation, a jump test will be carried out, measured with a force platform (FP4060-102000, Bertec Corporation). In this evaluation, subjects will warm up during 5 minutes on a treadmill, subsequently, they will perform dynamic stretches for the leg muscles. The jump test will be explained to participants and they will be asked to demonstrate how they will perform the assessment to ensure that they have understood it before going to the platform. Later, patients will go to the platform forces and will perform each jump three times (squat jump, Abalakov jump and countermovement jump test) with 60 seconds between jumps and 2 minutes between different jumps (table 1$).^{26-28}$ The maximum height of the jump will be analysed via the measurement of the flight time recorded on the force platforms, the eccentric power and the maximum concentric force performed. The Abalakov jump will be performed with the subject standing in an upright position with a full arm swing. A rapid downward movement will be immediately followed by a rapid upward vertical movement as high as possible, all in one sequence. The same procedure will be applied for the countermovement jump, however, this test will be performed with the hands on the hips to avoid arm swings. Finally, a squat jump will be performed with 90 degrees of flexion of the knee.

\section{Participant timeline}

The study design will be a double-blind randomised controlled trial. The flow chart of the trial is shown in figure 1 and the check list SPIRIT schedule is shown in figure 2 .

\section{Patient and public involvement}

Patients with PT were not involved in setting the research question or the outcome measures, however the concept

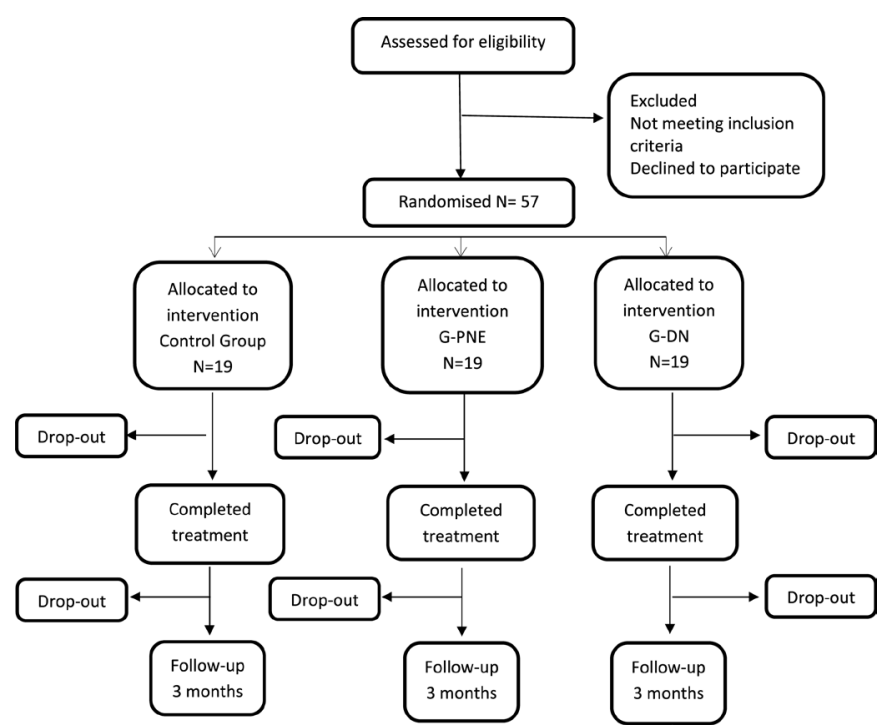

Figure 1 Flow diagram. Randomised controlled trial design. G-DN,dry needle group; G-PNE, percutaneous needle electrolysis group.

of patient involvement translated to the execution phases of the research. Patients and their families were central to the dissemination of the information, which helped to recruit study participants. We intend to disseminate the main results to trial participants and will seek patient and

\begin{tabular}{|c|c|c|c|c|c|c|}
\hline \multirow[b]{2}{*}{ TIMEPOINT** } & \multirow{2}{*}{$\begin{array}{c}\text { Enrolment } \\
-t_{1}\end{array}$} & \multirow{2}{*}{$\begin{array}{c}\text { Allocation } \\
0\end{array}$} & \multicolumn{2}{|c|}{ Close-out } & \multirow[b]{2}{*}{$t_{3}$} & \multirow[b]{2}{*}{$t_{4}$} \\
\hline & & & $t_{1}$ & $t_{2}$ & & \\
\hline \multicolumn{7}{|l|}{ ENROLMENT: } \\
\hline Eligibility screen & $x$ & & & & & \\
\hline Informed consent & $x$ & & & & & \\
\hline Allocation & & $x$ & & & & \\
\hline \multicolumn{7}{|l|}{ INTERVENTIONS: } \\
\hline \multicolumn{7}{|l|}{ Control group } \\
\hline \multicolumn{7}{|l|}{ G-PNE } \\
\hline \multicolumn{7}{|l|}{ G-DN } \\
\hline \multicolumn{7}{|l|}{ ASSESSMENTS: } \\
\hline $\begin{array}{r}\text { Baseline } \\
\text { demographic } \\
\text { information }\end{array}$ & $x$ & & & & & \\
\hline VISA-P & $x$ & & $x$ & & $x$ & $x$ \\
\hline VAS & & & $x$ & & $x$ & $x$ \\
\hline$S F-36$ & & & $x$ & & $x$ & $x$ \\
\hline Tendon structure US & & & $x$ & & $x$ & $x$ \\
\hline Jump test & & & $x$ & & $x$ & $x$ \\
\hline
\end{tabular}

Figure 2 Schedule for the enrolment and intervention. Schedule for enrolment and intervention per cluster. G-DN,dry needle group; G-PNE, percutaneous needle electrolysis group; -t1, baseline; $\mathrm{t1}-\mathrm{t} 2$, intervention period; T2, 8 weeks after baseline; $\mathrm{t} 3$, afterbaseline; T3, 10 weeks after baseline; t4, afterbaseline; T4, 3 months after baseline; US, ultrasound;VAS, Visual Analogue Scale. 
public involvement in the development of an appropriate method of dissemination.

\section{Sample size}

Regarding the sample size, a calculation of statistical power was made prior to the study. Accepting an alpha risk of 0.05 and a beta risk in a bilateral contrast, 19 subjects are needed in every treatment group to detect a difference equal or superior to 15 points on the VISA-P scale and assuming a SD of 15 points. ${ }^{29}$ The estimated rate of loss to follow-up is $20 \%$.

Recruitment of subjects for the trial will take place between October 2018 and March 2020 and will be carried out by means of informative campaigns targeted at different Sports Clubs and Federations by means of email and advertisements in the different University mass media.

The interested subjects will receive an email explaining the inclusion and exclusion criteria, as well as the purpose of the study. If they meet the defined criteria, they will be invited to send us their medical diagnosis.

\section{Recruitment}

Participants will be recruited from sports clubs by the physiotherapist or the coach. Contact has been made with various orthopaedists who will collaborate with recruitment, so that when they establish a diagnosis of this pathology in their examination room they can refer us to the patients for their recruitment to the study.

\section{Allocation}

Participants will be randomly assigned to either control group (CG) or dry needle group (DN-G) or PNE-G with a 1:1:1 allocation using an opaque envelope, with a block size of 15 participants (five for each group).

Sealed opaque randomisation envelopes with a studyspecific participant number will be supplied by an external statistician. A colleague not involved in the research study will take the sealed opaque numbered envelopes in order, by number and deliver the correct envelope to the treating physical therapist. The envelope will contain a piece of paper, which will be labelled with the same participant specific number, plus the group assignment (PNE-G, DN-G or CG).

Participants who fulfil the inclusion criteria will receive the standardised oral and written information, and, once they grant their consent to take part in the trial, they will be randomised into the three groups.

\section{Blinding}

Assessments regarding clinical recovery will be conducted by an assessor blinded to treatment allocation. Due to the nature of the intervention, participants can be blinded to allocation. Patients will be explained that they are going to receive a needling treatment, that it may be slightly painful and that if at any time they are unable to tolerate the pain they must inform the researcher to stop the intervention. In order to blind patients, all the interventions were made with the ultrasound and the
PNE device connected to simulate the same intervention in all groups. In contrast, the physiotherapist performing the intervention cannot be blinded, however he/she will be instructed not to disclose the allocation status of the participant at any time or during the follow-up assessments. An employee outside the research team will feed data into the computer in separate data sheets so that the researchers can analyse data without having access to information about the allocation.

With the intention of evaluating patient blinding, an online questionnaire will be sent to participants on completion of the study, asking them about the treatment they received.

\section{Data collection methods}

For the data collection, an oral questionnaire will be used containing questions targeted at collecting baseline data and information concerning the pathology.

Different questionnaires and assessment scales (VISA-P, VAS, SF-36) in Spanish will be given to each participant in paper when they attend the assessment, and they will be granted sufficient time to complete the same.

\section{Data management and statistical analysis}

In this study, all data will be entered electronically in the assessment room.

Original scales and questionnaires will be entered and kept on a locked file at the participating site.

Participant files are to be stored in numerical order and stored in a secure and accessible place and manner. Participant files will be maintained in storage for a period of 2 years after completion of the study.

The statistical analysis will be carried out by an intention-to-treat analysis. Variables will be described in number (percentage) and average (SD) or median (IQR) attending to their distribution. Quantitative variables will be analysed with the Shapiro-Wilk test in order to confirm their distribution and to determine correct statistical tests according to these results.

Outcomes will be analysed using mixed linear and logistic regression models considering participants as a random effect and group of treatment as fixed factors. Baseline characteristics will be introduced in the model as covariance. Numbers needed to treat index will also be calculated. The primary aim of the analysis will be to calculate the difference obtained in the VISA-P score after the intervention (final measurement - initial measurement). Finally, the magnitude of the effect of the result will be calculated and therefore its clinical importance, by means of the following formula:

$$
r=\sqrt{\frac{F(1, d f R)}{F(1, d f R)+d f R}}
$$

where $\mathrm{r}=$ Pearson's effect size correlation, $\mathrm{F}=\mathrm{F}$ test value, $\mathrm{dfR}=$ degree of freedom for $\mathrm{F}$ test value.

The significance level set for all the analysis will be $\mathrm{p} \leq 0.05$. 


\section{ETHICS AND DISSEMINATION}

The study design, procedures and informed consent procedure were approved and consequently the study will be carried out in compliance with the Helsinki Declaration of Human Rights. All participants will have to provide written Spanish informed consent (online supplementary appendix 1).

The results for this trial will be published in peer-reviewed international journals or otherwise made publicly available and will be presented at national and international conferences and symposiums irrespective of the outcomes.

Any modifications to the protocol, which may impact the study procedures, potential patient benefits or may affect patient safety, including changes of study objectives, study design, patient population, sample sizes, study procedures or significant administrative aspects will require a formal amendment to the protocol. Such amendments will be approved by the Ethics Committee prior to implementation and notified to the health authorities in accordance with local regulations.

All study-related information will be stored securely at the study site. All participant information will be stored in locked file cabinets in areas with limited access. All records that contain names or other personal identifiers, such as locator forms and informed consent forms, will be stored identified by code number. All local databases will be secured with password-protected access systems.

\section{Availability of data and material}

The data sets used and/or analysed during the current study are available from the corresponding author on reasonable request.

\section{DISCUSSION}

This study seeks to investigate the effects of physiotherapy needling techniques on pain, functionality and quality of life in PT.

PT is a common cause of knee pain in cases of degeneration of the patellar tendon. Among the causes of PT, extrinsic factors (eg, patellar tendon loading with exercise) and intrinsic factors (eg, malalignment, high patella, imbalances) have been proposed. ${ }^{30}$ Traditionally, the focus has been on quadriceps strengthening exercises and many reviews have shown that the effect of the treatment could be estimated to give the patients a $50 \%$ to $70 \%$ change of improvement on pain and functionality. ${ }^{6} 3132$

Regarding needling treatment, previous studies have shown a great improvement in PT using PNE in combination with EE, with all patients reporting an improvement at least 1 month after treatment. ${ }^{12-1418}$ This is an improvement compared with the minimum 3 months needed to improve symptoms by applying other conventional techniques (pharmacological and biological treatments, cold/heat techniques, shock waves, etc). Additionally, in a long-term study conducted in 2013, this technique was shown to improve symptoms quickly and steadily for at least 10 years. ${ }^{33}$ These findings demonstrate that this technique ensures that patients remain pain-free for a long period. Furthermore, we were only able to find four articles $^{12-1418}$ addressing the application of PNE for the recovery of PT, however, none of the articles studied were randomised controlled trials (RCTs), which entail limited evidence of the effectiveness of this technique.

In addition, there are no standardised protocols for the application of PNE, which explains the great variability in the number of sessions and application time based on the literature. Therefore, this study aims to facilitate clinical practice and combine the available methodology criteria in the application of this promising technique.

Regarding DN, the literature shows many similarities with PNE, since there is only one RCT that compares functionality improvements among patients who have received PRP. This study reflected that in the short-term PRP had better results for pain and functionality, however, DN was more effective than PRP after 6 months. ${ }^{34}$

For the application of both needling techniques, US-guidance is normally used to be able to observe first the presentation of the tendon, and later to observe the needle and enable a much more specific treatment approach. However, US has disadvantages including its operator dependence and the limited ability to rule out intra-articular disease. The sensitivity and specificity of ultrasonography for PT is between $58 \%$ and $94 \%$, respectively. $^{35}$

Moreover, functionality of the tendon is usually measured with the VISA-P, ${ }^{36}$ whereas jump tests (representing a similar action to that performed in subject's daily sports) are only evaluated in a few papers. ${ }^{2738}$ Countermovement jumps and squat jumps are the most reliable and valid field tests for the estimation of the explosive power of the lower limbs in physically active men. ${ }^{39}$ Thus, we will combine both, in order to be more accurate in the assessment of the tendon's functionality, and be able to assess changes that may affect their sport performance.

This study has several strengths. First, we will evaluate two techniques that currently lack strong evidence. However, in doing so, we are contributing to new knowledge in the field of the recovery of musculoskeletal injuries. Second, the role of invasive techniques will be determined by comparing the effects between these techniques and a control group. The reliability of data is ensured, as both patients and the assessor will be blinded. Third, a subanalysis with US will be performed to investigate changes in the presence of calcifications, cortical irregularities, neovascularisation, thickness, eco-intensity, eco-variation and eco-texture of the patellar tendon.

However, there are some limitations to this study. Blinding of the physiotherapist performing the intervention is not possible. Furthermore, follow-up is limited to 22 weeks after baseline.

The findings obtained may help advance the treatment of this injury by identifying the most effective treatment protocol and to avoid the associated consequences, such as the prevention of relapses and reducing the potential impact on the musculoskeletal system. 
Author affiliations

${ }^{1}$ iPhysio Research Group, Facultad de Ciencias de la Salud. Universidad San Jorge, Villanueva de Gallego, Aragón, Spain

${ }^{2}$ Departamento de Fisiatría y Enfermería, Facultad de Ciencias de la Salud, Universidad de Zaragoza, Zaragoza, Aragón, Spain

${ }^{3}$ Facultad de Ciencias de la Salud y del Deporte, Universidad de Zaragoza, Campus de Huesca, Aragón, Spain

${ }^{4}$ Kuwait Ministry of Health, Safat, Al Asimah, Kuwait

\section{Twitter Zaid Al-Boloushi @DrPT_Zaid}

Contributors MPL, EMG and PH conceived of the idea, and developed the intervention. MPL and PH wrote the article. MPL, MO, RMG, AVB, ZA, YH and PH developed the design of the trial. MO were involved in development of the statistical analysis of the trial and contributed to the content of the article. AVB contributed to the design and writing of the jump test protocol. All authors have read and approved the final manuscript.

Funding The authors have not declared a specific grant for this research from any funding agency in the public, commercial or not-for-profit sectors.

Competing interests None declared.

Patient consent for publication Not required

Provenance and peer review Not commissioned; externally peer reviewed.

Open access This is an open access article distributed in accordance with the Creative Commons Attribution Non Commercial (CC BY-NC 4.0) license, which permits others to distribute, remix, adapt, build upon this work non-commercially, and license their derivative works on different terms, provided the original work is properly cited, appropriate credit is given, any changes made indicated, and the use is non-commercial. See: http://creativecommons.org/licenses/by-nc/4.0/.

\section{ORCID iD}

Pablo Herrero http://orcid.org/0000-0002-9201-0120

\section{REFERENCES}

1 Knobloch K. The role of tendon microcirculation in Achilles and Patellar tendinopathy. J Orthop Surg Res 2008;3:18.

2 Zwerver J, Bredeweg SW, van den Akker-Scheek I. Prevalence of jumper's knee among nonelite athletes from different sports: a crosssectional survey. Am J Sports Med 2011;39:1984-8.

3 Stenroth L, Sefa S, Arokoski J, et al. Does magnetic resonance imaging provide superior reliability for Achilles and Patellar tendon cross-sectional area measurements compared with ultrasound imaging? Ultrasound Med Biol 2019;45:3186-98.

4 Taylor DW, Petrera M, Hendry M, et al. A systematic review of the use of platelet-rich plasma in sports medicine as a new treatment for tendon and ligament injuries. Clin J Sport Med 2011;21:344-52.

5 Andres BM, Murrell GAC. Treatment of tendinopathy: what works, what does not, and what is on the horizon. Clin Orthop Relat Res 2008;466:1539-54.

6 Larsson MEH, Käll I, Nilsson-Helander K. Treatment of Patellar tendinopathy - a systematic review of randomized controlled trials. Knee Surg Sports Traumatol Arthrosc 2012;20:1632-46.

7 Kongsgaard M, Kovanen V, Aagaard P, et al. Corticosteroid injections, eccentric decline squat training and heavy slow resistance training in patellar tendinopathy. Scand J Med Sci Sports 2009;19:790-802.

8 Murtaugh B, Ihm JM. Eccentric training for the treatment of tendinopathies. Curr Sports Med Rep 2013;12:175-82.

9 Young MA, Cook JL, Purdam CR. Eccentric decline squat protocol offers superior results at 12 months compared with traditional eccentric protocol for patellar tendinopathy in volleyball players. $\mathrm{Br} \mathrm{J}$ Sports Med 2005;39:102-5.

10 Ortega-Castillo M, Medina-Porqueres I. Effectiveness of the eccentric exercise therapy in physically active adults with symptomatic shoulder impingement or lateral epicondylar tendinopathy: a systematic review. J Sci Med Sport 2016;19:438-53.

11 James SLJ, Ali K, Pocock C, et al. Ultrasound guided dry needling and autologous blood injection for Patellar tendinosis * commentary. Br J Sports Med 2007;41:518-21.

12 Abat FDW, Gelber PE, Polidori F, et al. Effectiveness of the Intratissue percutaneous electrolysis (EPIR) technique and isoinertial eccentric exercise in the treatment of Patellar tendinopathy at two years follow-up. Muscles Ligaments Tendons J 2014;14:188-93.

13 Valera Garrido F, Minaya Muñoz F, SI JM. Effectiveness of electrolysis percutaneous intratisular $(\mathrm{EPI} 囚)$ in chronic insertional Patellar tendinopathy. Muscles Ligaments Tendons J 2010;21:227-36.
14 Sánchez-lbáñez JM, Alves R, Polidori F, et al. Effectiveness of ultrasound-guided percutaneous electrolysis intratendon (Epl) in the treatment of insertional Patellar tendinopathy in soccer players. $\mathrm{Br} \mathrm{J}$ Sports Med 2013;47:e2.17-e2.

15 Zwerver J, Verhagen E, Hartgens F, et al. The TOPGAME-study: effectiveness of extracorporeal shockwave therapy in jumping athletes with Patellar tendinopathy. design of a randomised controlled trial. BMC Musculoskelet Disord 2010;11:6.

16 Riggin CN, Chen M, Gordon JA, et al. Ultrasound-Guided dry Needling of the healthy rat supraspinatus tendon elicits early healing without causing permanent damage. J Orthop Res 2019;37:2035-42.

17 Krey D, Borchers J, McCamey K. Tendon needling for treatment of tendinopathy: a systematic review. Phys Sportsmed 2015;43:80-6.

18 Abat F, Valles S-L, Gelber P-E, et al. An experimental study of muscular injury repair in a mouse model of notexin-induced lesion with EPI® technique. BMC Sports Sci Med Rehabil 2015;7:7.

19 Valera-Garrido F, Minaya-Muñoz F, Sánchez-lbáñez JM, et al. Comparison of the acute inflammatory response and proliferation of dry needling and electrolysis percutaneous intratissue (Epl) in healthy rat Achilles tendons. Br J Sports Med 2013;47:e2.52-e2.

20 Alfredson H, Pietilä T, Jonsson P, et al. Heavy-load eccentric calf muscle training for the treatment of chronic Achilles tendinosis. Am J Sports Med 1998;26:360-6.

21 Grävare Silbernagel K, Crossley KM. A proposed Return-to-Sport program for patients with Midportion Achilles tendinopathy: rationale and implementation. J Orthop Sports Phys Ther 2015;45:876-86.

22 Padrón-Benítez A, Rojas-Mederos S. A comparative study between low and high intensity percutaneous needle electrolysis in patients with Patellar tendinopathy: a structural and functional analysis. J Invasive Tech Phys Ther 2016;1:10-17.

23 Hernandez-Sanchez S, Hidalgo MD, Gomez A. Cross-Cultural adaptation of VISA-P score for Patellar tendinopathy in Spanish population. J Orthop Sports Phys Ther 2011;41:581-91.

24 Alonso J, Prieto L, Anto JM. The Spanish version of the SF-36 health survey- a measure of clinical outcomes. Medicina Clinica 1995;104:771-6.

25 Martinoli C. Musculoskeletal ultrasound: technical guidelines. Insights Imaging 2010;1:99-141.

26 Rodríguez-Rosell D, Mora-Custodio R, Franco-Márquez F, et al. Traditional vs. Sport-Specific vertical jump tests: reliability, validity, and relationship with the legs strength and sprint performance in adult and teen soccer and Basketball players. J Strength Cond Res 2017;31:196-206.

27 Helland C, Bojsen-Møller J, Raastad T, et al. Mechanical properties of the patellar tendon in elite volleyball players with and without patellar tendinopathy. Br J Sports Med 2013;47:862-8.

28 Gual G, Fort-Vanmeerhaeghe A, Romero-Rodríguez D, et al. Effects of In-Season inertial resistance training with eccentric overload in a sports population at risk for Patellar tendinopathy. J Strength Cond Res 2016;30:1834-42.

29 Scott A, LaPrade RF, Harmon KG, et al. Platelet-Rich plasma for Patellar tendinopathy: a randomized controlled trial of LeukocyteRich PRP or Leukocyte-Poor PRP versus saline. Am J Sports Med 2019;363546519837954

30 Figueroa D, Figueroa F, Calvo R. Patellar tendinopathy: diagnosis and treatment. J Am Acad Orthop Surg 2016;24:e184-92.

31 Lian Østein B., Engebretsen L, Bahr R. Prevalence of jumper's knee among elite athletes from different sports: a cross-sectional study. Am J Sports Med 2005;33:561-7.

32 Visnes $\mathrm{H}$, Bahr R. The evolution of eccentric training as treatment for patellar tendinopathy (jumper's knee): a critical review of exercise programmes. Br J Sports Med 2007;41:217-23.

33 Abat F, Gelber PE, Polidori F, et al. Clinical results after ultrasoundguided intratissue percutaneous electrolysis (EPI尺) and eccentric exercise in the treatment of Patellar tendinopathy. Knee Surg Sports Traumatol Arthrosc 2015;23:1046-52.

34 Dragoo JL, Wasterlain AS, Braun HJ, et al. Platelet-Rich plasma as a treatment for Patellar tendinopathy: a double-blind, randomized controlled trial. Am J Sports Med 2014;42:610-8.

35 Warden SJ, Kiss ZS, Malara FA, et al. Comparative accuracy of magnetic resonance imaging and ultrasonography in confirming clinically diagnosed Patellar tendinopathy. Am J Sports Med 2007;35:427-36.

36 Hernandez-Sanchez S, Hidalgo MD, Gomez A. Cross-cultural adaptation of VISA-P score for patellar tendinopathy in Spanish population. J Orthop Sports Phys Ther 2011;41:581-91.

37 Andriolo L, Altamura SA, Reale D, et al. Nonsurgical treatments of Patellar tendinopathy: multiple injections of platelet-rich plasma are a suitable option: a systematic review and meta-analysis. Am J Sports Med 2019;47:1001-18. 
38 de Vries A, Zwerver J, Diercks R, et al. Effect of patellar strap and sports tape on pain in patellar tendinopathy: a randomized controlled trial. Scand J Med Sci Sports 2016;26:1217-24.
39 Markovic G, Dizdar D, Jukic I, et al. Reliability and factorial validity of squat and countermovement jump tests. $J$ Strength Cond Res 2004; 18:551-5. 